\title{
Hybrid Approach Combining Multiple Characterization Techniques and Simulations for Microstructural Analysis of Proton Exchange Membrane Fuel Cell Electrodes
}

\author{
Firat C. Cetinbas, ${ }^{* a}$ Rajesh K. Ahluwalia, ${ }^{a}$ Nancy Kariuki, ${ }^{b}$ Vincent De Andrade, ${ }^{c}$ Dash Fongalland ${ }^{d}$, Linda Smith ${ }^{d}$, \\ Jonathan Sharman, ${ }^{\mathrm{d}}$ Paulo Ferreira, ${ }^{\mathrm{e}}$ Somaye Rasouli ${ }^{\mathrm{e}}$ and Deborah J. Myers ${ }^{\mathrm{b}}$ \\ ${ }^{a}$ Argonne National Laboratory, Nuclear Engineering Division, IL 60439, USA \\ ${ }^{\mathrm{b}}$ Argonne National Laboratory, Chemical Sciences and Engineering Division, IL 60439, USA \\ ${ }^{\mathrm{c}}$ Argonne National Laboratory, Advanced Photon Source, IL 60439, USA \\ ${ }^{\mathrm{d} J o h n s o n ~ M a t t h e y, ~ L o n d o n ~ E C 4 A ~ 4 A B, ~ U K ~}$ \\ ${ }^{\mathrm{e}}$ University of Texas at Austin, Mechanical Engineering, TX 78712-1591, USA
}

\begin{abstract}
The cost and performance of proton exchange membrane fuel cells strongly depend on the cathode electrode due to usage of expensive platinum (Pt) group metal catalyst and sluggish reaction kinetics. Development of low Pt content high performance cathodes requires comprehensive understanding of the electrode microstructure. In this study, a new approach is presented to characterize the detailed cathode electrode microstructure from $\mathrm{nm}$ to $\mu \mathrm{m}$ length scales by combining information from different experimental techniques. In this context, nanoscale X-ray computed tomography (nano-CT) is performed to extract the secondary pore space of the electrode. Transmission electron microscopy (TEM) is employed to determine primary C particle and Pt particle size distributions. X-ray scattering, with its ability to provide size distributions of orders of magnitude more particles than TEM, is used to confirm the TEMdetermined size distributions. The number of primary pores that cannot be resolved by nano-CT is approximated using mercury intrusion porosimetry. An algorithm is developed to incorporate all these experimental data in one geometric representation. Upon validation of pore size distribution against gas adsorption and mercury intrusion porosimetry data, reconstructed
\end{abstract}

\footnotetext{
*corresponding author: ccetinbas@anl.gov
} 
ionomer size distribution is reported. In addition, transport related characteristics and effective properties are computed by performing simulations on the hybrid microstructure.

Keywords: PEMFC electrode, catalyst layer, electrode microstructure, X-ray computed tomography, effective transport properties

\section{Introduction}

Proton exchange membrane fuel cell (PEMFC) technology is a promising alternative to replace fossil fuel-dependent internal combustion engines (ICEs) for automotive propulsion power. In order to be competitive in the market, the cost and lifetime of PEMFC systems must be within close proximity of those of ICEs. The ultimate U.S. Department of Energy (DOE) target is to decrease the cost of an automotive PEMFC system from $\$ 55 / \mathrm{kW}$ to $\$ 30 / \mathrm{kW}$, and to increase the durability from $\sim 3900$ to $>5000$ hours [1]. The breakdown of the overall cost for an automotive fuel cell stack shows that the Pt group metal (PGM) catalyst is the major cost-driver [1]. Besides developing PGM-free catalysts, the main strategy in decreasing the fuel cell cost is to lower the amount of PGM without sacrificing performance.

A typical PGM electrode consists of a carbon (C)-supported Pt catalyst, perfluorosulfonic acid ionomer binder, pore space, and under certain conditions, liquid water. The $\mathrm{C}, \mathrm{Pt}$, and ionomer binder form agglomerates as illustrated in Fig. 1. This agglomeration results in large pores (secondary pores) between the agglomerates and small pores (primary pores) inside them. The electrochemical reactions occur at the triple phase $(\mathrm{C}, \mathrm{Pt}$, ionomer/water) boundaries where electrons $\left(\mathrm{e}^{-}\right)$, protons $\left(\mathrm{H}^{+}\right)$, and reactants meet at the Pt catalyst surface. Within this complex geometrical and chemical environment, $\mathrm{C}$ support and ionomer binder create networks for 
conduction of $\mathrm{e}^{-}$and $\mathrm{H}^{+}$, respectively, and the pore space generates pathways for reactant transport and product removal, as illustrated in Fig. 1.

Literature presents many studies reporting either an optimum ionomer [2-7] or Pt loading $[4,6]$ but determination of an optimum electrode composition is quite challenging. The electrode microstructure and its effects on fuel cell performance are not as trivial as the functions of constituents, because various components and transport processes influence each other. Therefore, a comprehensive insight into electrode microstructure and its interactions with the corresponding transport mechanisms is required to improve the performance of the electrodes under a variety of conditions and also to lower the amount of PGM catalyst without minimal loss in performance. This insight will also establish a foundation for determining the effects of the variables such as ionomer content, catalyst-ionomer ink solvent, and catalyst type (e.g., Pt alloy versus $\mathrm{Pt}$ ) on the microstructure, transport properties, and resulting electrode performance.

There are several analysis techniques to investigate the PEMFC electrode microstructure. Mercury intrusion porosimetry (MIP) and Brunauer-Emmett-Teller (BET) gas adsorption porosimetry are the commonly used methods for evaluation of pore sizes and specific surface areas of porous materials such as PEMFC catalysts and electrodes. Gas adsorption porosimetry is preferred over MIP for measuring smaller pores because the high intrusion pressure of mercury may be destructive for the microstructure, and MIP is not successful at resolving pores smaller than $3 \mathrm{~nm}$. Uchida et al. [2] were one of the first to report the bimodal pore size distributions corresponding to primary and secondary pores by using MIP. They also showed that the pore sizes are strongly dependent on the type of C support. In another study, Sobolyeva et al. [8] studied the microstructures of $\mathrm{C}$ supports by employing the nitrogen gas adsorption technique. It is important to note that both MIP and BET methods rely on the calculation of pore sizes based 
on surface tension, capillary forces, and pressure. Therefore, these approaches are limited to provide only bulk property data, whereas the anisotropic heterogeneous electrode microstructure requires more detailed quantification.

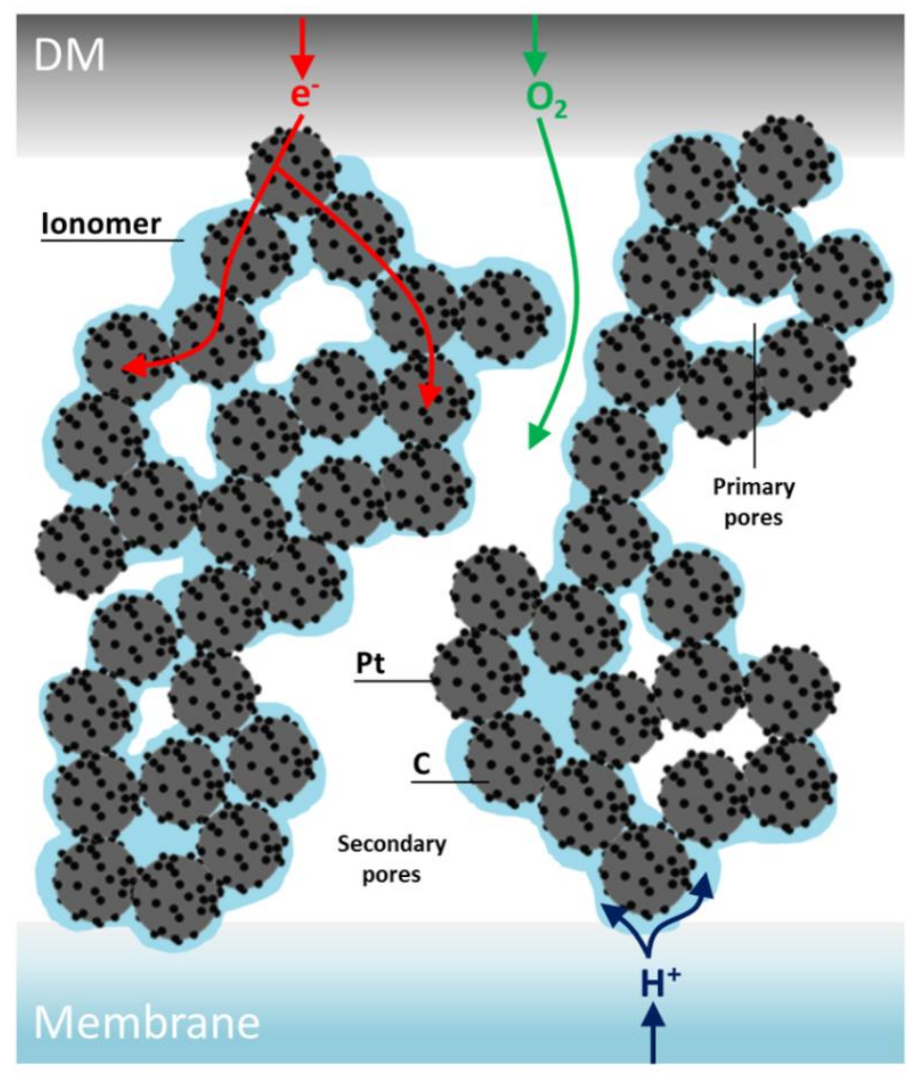

Figure 1. Representation of the principal components of the cathode electrode and their functions

Besides the standard porosimetry methods, techniques that can capture the internal microstructure are critical in gaining insight into the three-dimensional distribution of the electrode components. The two-dimensional electron microscopy techniques, scanning electron microscopy (SEM) and transmission electron microscopy (TEM), are popular tools for characterization of PEMFC electrodes [9]. SEM is commonly applied to resolve macro structures while TEM [10-14], with its high resolution $(<1 \mathrm{~nm})$, serves as a powerful tool to characterize size, shape, distribution and crystalline structure of $\mathrm{C}$ supported nano-scale catalyst particles in the electrode. Besides the catalyst and support characterization, using atomic force microscopy 
(AFM) and TEM, Xie et al. [15] showed that the ionomer content changes the sizes of C aggregates and the thickness of the ionomer film covering the $\mathrm{C}$ surface. In addition, recently advanced TEM techniques such as aberration-corrected scanning transmission electron microscopy (STEM) with energy-dispersive spectroscopy (EDS) [16], and electron tomography (ET) [17], coupled with cesium ion exchange, were able to provide information about the microstructure of the ionomer phase in the electrode.

The two-dimensional electron microscopy images are the representations of threedimensional nanostructures. To be able to investigate the whole electrode with TEM, 100-150 $\mathrm{nm}$ thick slices need to be extracted from the electrode $[5,9]$. However, slicing limits the spatial integrity between consecutive images and this method suffers from not being fully representative of the three-dimensional microstructure, i.e. the porosity is generally underestimated [18]. Electron tomography allows the observation of three-dimensional nanostructures. In this technique, two-dimensional images of the sample are taken at each angle step (i.e. between $-70^{\circ}$ to $+70^{\circ}$, separated by one degree) as it rotates; these are then aligned and reconstructed into a three-dimensional image sequence by using various numerical algorithms. Lopez-Haro et al. [17] employed ET and investigated the ionomer coverage on the $\mathrm{C}$ support for different compositions. They determined that a $7 \mathrm{~nm}$ film of ionomer is formed on the carbon with the coverage of ionomer on the carbon increasing with increasing ionomer content. This technique is quite successful in providing detailed, high-resolution three-dimensional images of nanostructures of $\mathrm{C}, \mathrm{Pt}$, and ionomer by focusing on a few $\mathrm{C}$ particles. While ET provides important information regarding the ionomer-carbon interface, in the modeling of electrode transport properties it is important to determine the nano- and microstructure over the larger length scales typical of 
PEMFC electrode thickness $(10-15 \mu \mathrm{m})$. In addition, considering the heterogeneous morphology of the electrode, this type of ET results may not be representative of the whole electrode.

Focused ion beam-scanning electron microscopy (FIB-SEM) is a commonly-used technique for three-dimensional quantification of the electrode microstructure. In this method, FIB operates as a milling tool and extracts a series of sections from the sample, which are subsequently characterized by SEM. The captured images are reconstructed into a threedimensional representation of the electrode. This technique has anisotropic resolution, so the high in-plane resolution of SEM is bounded by the sensitivity of the milling operation in the cutting direction. Thiele et al. $[19,20]$ used the FIB-SEM technique to image the microstructure of the PEMFC electrode with $30 \mathrm{~nm}$ increments of serial-sectioning and $5 \mathrm{~nm}$ resolution for SEM imaging. They described the whole reconstruction procedure in detail and reported pore size distributions for the electrode microstructure. Recently, they [21] addressed the lack of contrast between pores and catalyst layer material and tried to overcome this problem by filling the pores with $\mathrm{ZnO}$. It is worth mentioning that the application of FIB-SEM technique to PEMFC electrodes is limited by the vacuum environment requirement and its destructive nature.

Nano-scale X-ray computed tomography (nano-CT) offers non-destructive quantification of three-dimensional microstructures. In this technique, an X-ray beam passes through the sample and the transmitted beam is recorded as the sample is rotated between 0 and $180^{\circ}$. The recorded projection images are reconstructed into the three-dimensional electrode microstructure. Employing nano-CT with $32.5 \mathrm{~nm}$ voxel size, Epting et al. [18] studied the morphology of PGMbased electrodes. They reported volumetric size distributions of secondary pores (larger than $32.5 \mathrm{~nm})$ and solid phase $(\mathrm{C}, \mathrm{Pt}$, ionomer and primary pores corresponds to agglomerates) size distributions. Litster et al. [22] used the same technique to study the morphological and transport 
properties of the electrodes. They calculated effective reactant diffusivities based on Fick's law and numerical solution of the diffusion equation in the extracted pore volume from X-ray data. Application of this technique is limited by the resolution and the presence of low atomic number materials in the electrode microstructure. The electrode nano-structural features, such as 2-10 nm catalyst particles and primary pores, cannot be disseminated from the nano-CT images because of the low resolution, i.e., $20 \mathrm{~nm}$. Furthermore, the similar atomic numbers of the constituent materials result in insufficient contrast in the intensity map and makes it quite challenging to distinguish $\mathrm{C}$ and ionomer. State-of-the-art nano-CT provides three-dimensional images of the secondary pore (pores larger than resolution) morphology, whereas the details of primary pores, C, Pt, and ionomer are mingled within the solid volume. Recently, Babu et al. [23] ion exchanged the protons in Nafion ${ }^{\circledR}$ with cesium $\left(\mathrm{Cs}^{+}\right)$and imaged the ionomer in thick PGM-free electrodes using absorption contrast nano-CT. Even though the state-of-the-art resolution of nano-CT is rather low, unlike FIB-SEM it doesn't require a vacuum environment. This allows nano-CT to work under various operating conditions i.e. it was applied to solid oxide fuel cell (SOFC) electrodes at operating temperatures of $700-850^{\circ} \mathrm{C}$ with a special stage design [24].

Besides the aforementioned experimental methods, numerous numerical studies [25-37] have been conducted to investigate effect of electrode microstructure on the performance and transport properties. Many of them [25-30] assumed an ideal agglomerate microstructure and applied it to macroscale transport models with various techniques, while others [31-37] focused on pore scale phenomenon by numerically generating electrode microstructures. In these studies, the electrode microstructure was numerically regenerated with various techniques such as Gaussian random field method [31, 32], random sphere packing [33-35], and statistical generation of distinct phases within a regular grid [36-37]. Using Gaussian random field method, 
secondary pore network of electrode layer was reconstructed with $100 \mathrm{~nm}$ voxels based on the two-point correlation function that they obtained from TEM images [31, 32]. They considered C, Pt and ionomer as a mixture while pores smaller than $100 \mathrm{~nm}$ are neglected. Other work [33-35] represented the electrode as randomly-packed $\mathrm{C}$ spheres surrounded by a constant thickness ideal ionomer film and neglected the existence of Pt particles. Kim and Pitsch [33] employed simulated-annealing technique and $60 \mathrm{~nm}$ spheres were randomly moved until the desired porosity was obtained. Similarly, Lange et al. $[34,35]$ randomly packed C spheres by using rules regarding placement of each new $\mathrm{C}$ particle and a specified overlapping tolerance to reconstruct the electrode structure. Zhang et al. [36] reconstructed the electrode from two phases; $50 \mathrm{~nm}$ voxels representing $\mathrm{C}$ supported $\mathrm{Pt}$ particles and ionomer-pore mixture. Siddique et al. [37] generated all the nanostructures in the electrode, including the Pt phase, based on growing agglomerates of C cells with the help of a quartet structure generation set (QSGS) algorithm [38].

To the best of our knowledge, neither nano-CT nor FIB-SEM was successful in separately resolving individual $\mathrm{C}, \mathrm{Pt}$ particles, or ionomer within the overall three-dimensional electrode morphology. Individual components of the electrode microstructure can be generated numerically as in Siddique and Liu's study [37], however the random nature of this method lacks experimental support and validation. In this study, an innovative technique is introduced for microstructure characterization. This technique combines the pore morphology from nano-CT data with USAXS, TEM and porosimetry data by employing a numerical algorithm to reconstruct detailed electrode microstructure (including $\mathrm{C}, \mathrm{Pt}$, and ionomer phases). Unlike aforementioned numerical models, experimentally-measured size distributions of the carbon primary particles and the catalyst nanoparticles are taken into account and these particles are 
placed in a morphology obtained directly from the nano-CT. This hybrid experimental-numerical method offers to approximate the primary pores, ionomer microstructure, $\mathrm{C}$ and Pt particles that are all not available from the nano-CT data alone. In this context, nano-CT is used to extract the three-dimensional morphology of the cathode. A series of TEM images were taken to analyze the size distributions of $\mathrm{C}$ and $\mathrm{Pt}$ particles. In order to compare global applicability of the TEM analysis, ultra-small angle and small-angle synchrotron X-ray scattering (USAXS and SAXS) were used to determine the $\mathrm{C}$ and $\mathrm{Pt}$ particle size distributions, respectively. The porosimetry data was used to approximately determine the amount of primary pores not captured in the nanoCT analysis. Combining all the experimental data, individual C, Pt and ionomer phases were discretely regenerated with the numerical algorithm introduced in this study. The resulting structure is validated by comparing the pore size distributions against MIP and BET techniques. Subsequently, to demonstrate the capabilities of the model in determining charge and reactant transport properties, simulations were conducted for various sub-volumes with different porosities.

\section{Hybrid Approach Methodology}

The hybrid approach combines the strengths of the aforementioned microstructure characterization techniques within one geometric representation and resolves the electrode nanostructures in various length scales. The general approach can be described as the following: three-dimensional secondary pore morphology and the solid phase (unresolved mixture of $\mathrm{Pt}, \mathrm{C}$, ionomer and primary pores) geometry are extracted by use of nano-CT. Then, based on a numerical algorithm, which uses TEM, X-ray scattering, and porosimetry data as input and is described in detail in section 2.3, the C network, Pt catalyst particles, and ionomer phase are 
reconstructed into the solid geometry obtained from the nano-CT analysis. The employed experimental characterization techniques and the data obtained from them are listed in Table 1.

Table 1. Experimental characterization techniques and the obtained data

\begin{tabular}{ll}
\hline Technique & Data \\
\hline Nano-CT & Pore network with $20 \mathrm{~nm}$ voxel size \\
TEM & Size distribution of primary C and Pt particles \\
& Complemantary data for size distribution of \\
USAXS & primary C and Pt particles \\
MIP & Pore volume size distribution $(3 \mathrm{~nm}-1 \mu \mathrm{m})$ \\
BET & Pore volume size distribution $(1 \mathrm{~nm}-100 \mathrm{~nm})$ \\
\hline
\end{tabular}

The catalyst used in this study was prepared by Johnson Matthey Fuel Cells (JMFC) and consists of heat-treated 29.7 wt\% Pt nanoparticles supported on an Akzo Nobel Ketjen EC300J carbon black $(\mathrm{Pt} / \mathrm{C})$ with a metal surface area of $37 \mathrm{~m}^{2} / \mathrm{g}-\mathrm{Pt}$, as determined using gas-phase CO adsorption, and a number mean diameter of $5.42 \pm 1.78 \mathrm{~nm}$, as determined using TEM image analysis, and a number mean diameter of $5.07 \mathrm{~nm}$ as determined using small angle X-ray scattering. The as-prepared $\mathrm{Pt} / \mathrm{C}$ catalyst had a mean Pt diameter of $\sim 2 \mathrm{~nm}$ and was heat treated to obtain a mean diameter similar to that typically observed for alloy catalysts, as the motivation for developing this methodology was to determine the influence of using Pt alloy versus Pt nanoparticle catalysts on the cathode structure and transport properties. A catalystperfluorosulfonic acid ionomer layer supported on a polytetrafluoroethylene (PTFE) sheet was prepared by JMFC from an aqueous-based catalyst-ionomer ink with an ionomer to carbon weight ratio of 0.8 . 


\subsection{Nano Scale X-ray Computed Tomography}

The X-ray radiographs were acquired using the Xradia nano XCT-S100 TXM at beam line 32-ID-C at the Advanced Photon Source, Argonne National Laboratory. The data were acquired at $8 \mathrm{keV}$ in Zernike phase contrast mode using a Fresnel zone plate with a $60 \mathrm{~nm}$ outermost zone width. 720 images were acquired over $180^{\circ}$ rotation with an exposure time of 2 seconds.

The dried catalyst-ionomer layer was removed from the PTFE sheet using a scalpel and sectioned into flakes with the following approximate dimensions: $50 \mu \mathrm{m}$ long, $10 \mu \mathrm{m}$ wide, and $10 \mu \mathrm{m}$ thick. These flakes were mounted on the end of a tomography needle using a quicksetting epoxy and the needle-supported samples were mounted on an air bearing stage in the beam path.

The projection images were reconstructed into a 3D image sequence with $20 \mathrm{~nm}$ voxel size using the software Tomopy (http://www.aps.anl.gov/tomopy/), an open source Python based toolbox for the analysis of synchrotron tomographic data. To be used in the hybrid approach, a 1 $\mu \mathrm{m} \times 1 \mu \mathrm{m}$ x $1 \mu \mathrm{m}$ volume was cropped from the reconstructed X-ray image as shown in Fig. 2a. Subsequently, histogram equalization and normalization were applied to the extracted volume in order to enhance the contrast. As illustrated in Fig. 2a, the image sequence was binarized into solid and pore spaces by applying a manual threshold. In addition, the pore sizes were calculated by employing the average chord length method [15]. In agreement with the literature [15, 17] and the MIP data, the mode of the pore size distribution derived in this manner (Fig. 2b) is approximately $200 \mathrm{~nm}$. It should be recalled that the pores smaller than $20 \mathrm{~nm}$ are not captured by the nano-CT technique. 


\subsection{Particle Size Distribution Analysis by TEM and X-ray Scattering}

\section{TEM}

The catalyst powder was dispersed in ethanol, ultrasonicated, and deposited on a carbon/copper grid for TEM characterization of Pt particles size. The presence of Pt particles on the support complicated the analysis to extract the primary carbon size from the TEM images. To address this issue, a non-catalyzed carbon support subjected to the same heat treatment protocol as the catalyst powder was used to determine the primary carbon particle size and prepared for TEM characterization using the same deposition procedure. The size of the $\mathrm{Pt}$ particles and the carbon support primary particle size were observed using a JEOL 2010F TEM operated at $200 \mathrm{kV}$. The sizes of two hundred $\mathrm{Pt}$ and two hundred primary carbon particles were analyzed from a series of bright-field TEM micrographs. The equivalent diameters of the particles were determined by using Image $\mathbf{J}$ software to calculate the projected area of the individual particles and converting this to the equivalent diameter of a spherical particle with the same projected area.

The resulting primary carbon particle size distribution is presented along with one of the TEM images in Figs. $2 \mathrm{c}$ and $2 \mathrm{~d}$. A sample image that is used for Pt size calculation and the resulting Pt particle size distributions are presented in Figs. 2e and 2f.

\section{$X$-ray Scattering}

The heat-treated carbon powder and the catalyst powder were mounted in holes of $9 \mathrm{~mm}$ diameter in a $0.1 \mathrm{~mm}$ thick paper which were sealed with $\mathrm{Scotch}^{\mathrm{TM}}$ tape. The samples were then supported in a custom-made mount. X-ray scattering in the ultra-small angle (USAXS) to smallangle (SAXS) regions $\left(\mathrm{Q}=1.2 \times 10^{-4}\right.$ to $\left.0.28 \AA^{-1}\right)$ was performed at beam line 9-ID-C at the 
Advanced Photon Source at Argonne National Laboratory using an X-ray energy of $12 \mathrm{keV}$, an advanced-design Bonse-Hart camera (USAXS) with an integrated fixed length pinhole SAXS camera (pinSAXS) [39].

The USAXS data for the carbon powder and SAXS data for the catalyst powder were analyzed using Irena, a suite of macros written for the Igor Pro software platform [40]. The scattering data were fitted with a log-normal distribution in the 0.02 to $0.28 \AA^{-1} \mathrm{Q}$ range to obtain Pt particle size distribution. The scattering data in the $2 \times 10^{-4}$ to $0.02 \AA^{-1} \mathrm{Q}$ range were also fitted with a log-normal distribution to obtain the carbon particle size distribution. The resulting primary carbon particle size distribution is presented along with that obtained from TEM in Fig. 2d. The resulting Pt particle size distribution is presented along with that obtained from TEM in Fig. 2f. These figures show that the TEM and USAXS/SAXS-determined particle size distributions are in agreement, validating the TEM-determined particle size distributions which utilize a small number of particles (200) relative to that analyzed via USAXS/SAXS $\left(\sim 10^{14}\right)$ and in the overall catalyst layer sample. 
a)

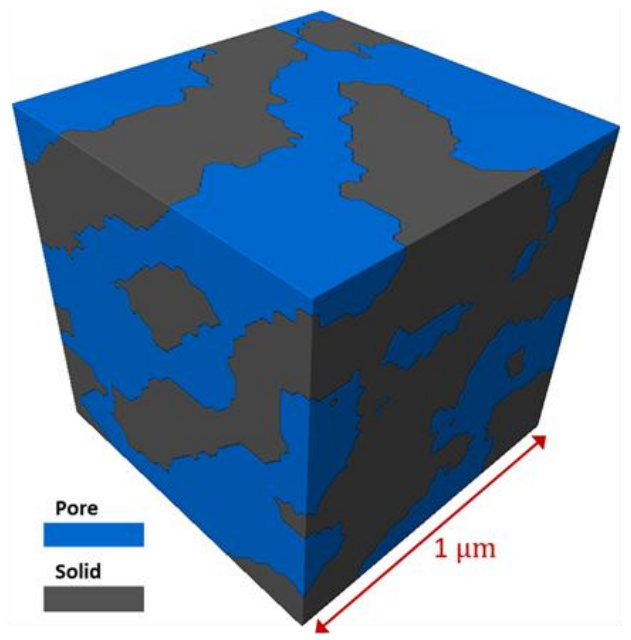

c)

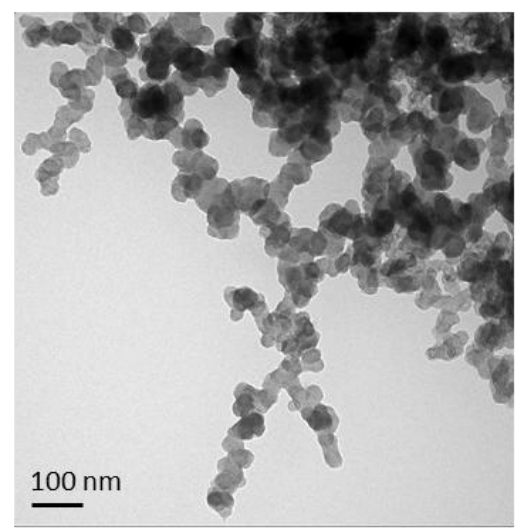

e)

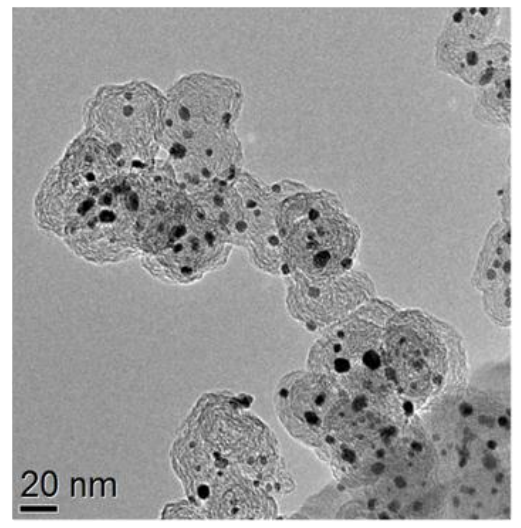

b)

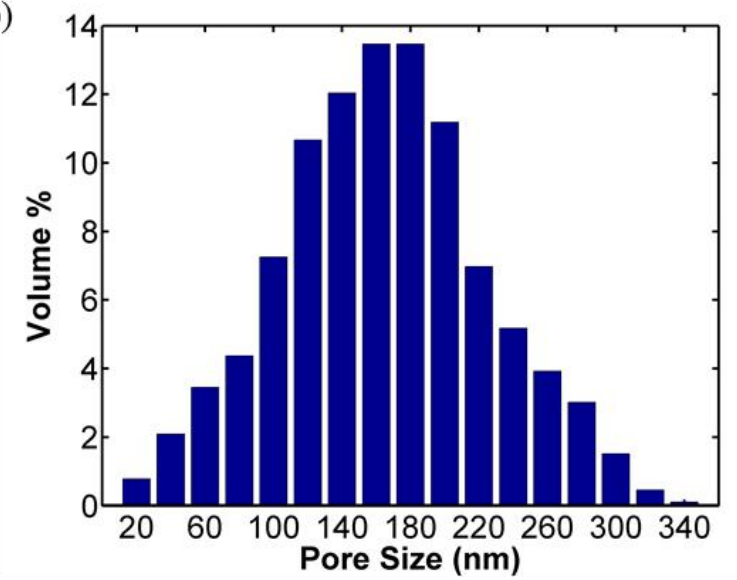

d)

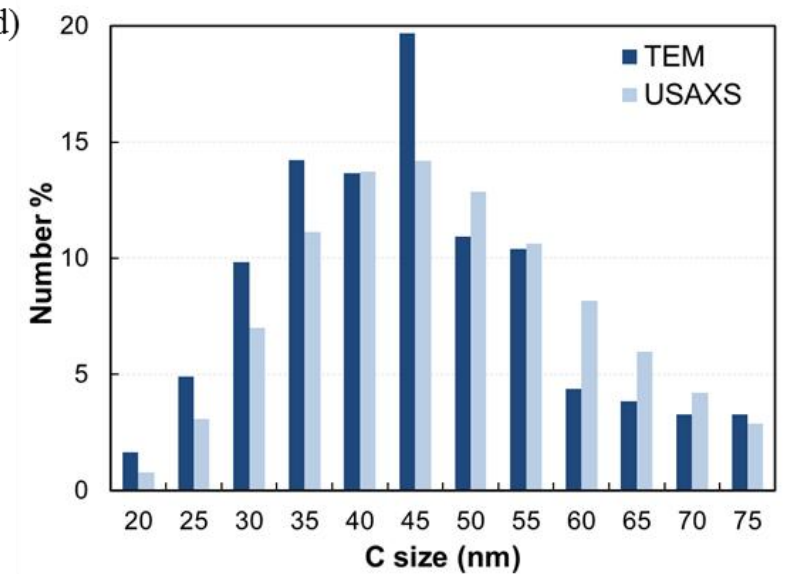

f)



Figure 2. (a) Nano-CT volume after segmentation to pore (void) and the solid phase $(\mathrm{C}, \mathrm{Pt}$, ionomer) to be used in the hybrid approach; (b) Corresponding pore size distribution; (c) TEM image of annealed Ketjen 300J C in powder; (d) Size distribution of primary C particles by TEM and USAXS; (e) TEM image of Pt on annealed C; (f) Size distribution of Pt particles from SAXS and TEM image analysis. The inset shows the size distribution of Pt particles from TEM image analysis binned to size of the modeling grid 


\subsection{Porosimetry}

The pore size distribution in the catalyst layer was measured by $\mathrm{N}_{2}$ adsorption BET and MIP techniques. $\mathrm{N}_{2}$ adsorption was performed at $77 \mathrm{~K}$ with a Micromeritics ASAP2020 surface area and pore size analyzer. Pore sizes up to $100 \mathrm{~nm}$ were determined from the measured pressure by using the Kelvin equation.

$$
\ln \left(\frac{P}{P_{0}}\right)=-\frac{2 \gamma V_{L}}{R T} \frac{1}{r_{m}}
$$

where $P$ is the pressure, $P_{0}$ is the saturation pressure, $\gamma$ is the surface tension, $V_{L}$ is the molar volume of the liquid and $r_{m}$ is the pore radius based on capillary assumption.

A Micromeritics Autopore IV 9520 was used to perform mercury intrusion porosimetry at pressures up to $60 \mathrm{kpsia}$. The meso and macro pore sizes up to $1 \mu \mathrm{m}$ were calculated using the Washburn equation, which allows $r_{m}$ to be calculated from the applied pressure of the nonwetting working fluid mercury.

$$
P_{a}=-\frac{2 \gamma \cos \theta}{r_{m}}
$$

where $P_{a}$ is the applied pressure, and $\theta$ is the contact angle.

\subsection{Numerical Algorithm}

Overall the geometry of the solid phase (corresponding to $\mathrm{C}, \mathrm{Pt}$, ionomer and primary pores) and the secondary pores are obtained from the nano-CT analysis as shown in Fig. 2a. The geometric (shape, size, orientation) and topological (connectivity) properties of the nanostructures within the nano-CT data are yet to be resolved. In this section, an algorithm is presented to regenerate the solid phase in the nano-CT data using the experimentally-determined 
$\mathrm{C}$ and Pt particle size distributions and volume fractions, the primary pore size, and the ionomer volume fraction.

The electrode microstructure strongly depends on the ink composition and the manufacturing techniques. Therefore, the volume fraction of each phase needs to be determined accurately prior to reconstruction. The composition of nano-CT solid volume can be calculated based on the manufacturer's specifications, such as Pt to $\mathrm{C}$ weight ratio $(f)$ and ionomer to $\mathrm{C}$ weight ratio $\left(f_{I C}\right)$, however the volume fraction of primary pores within the solid volume must be determined first. In this context, MIP data is used to calculate the volume of pores smaller than the X-CT data voxel size $(20 \mathrm{~nm})$. It is deduced that $7.18 \%$ of the total pore volume is from pores smaller than $20 \mathrm{~nm}$ and assumed that this ratio is constant for each subsample extracted from nano-CT data. Based on these assumptions, the volume fraction of the primary pores $\left(\varepsilon_{v p}\right)$ and other phases are calculated as following:

$$
\begin{gathered}
\varepsilon_{v p}=\varepsilon_{x r} \frac{f_{p m}}{1-f_{p m}} \\
\varepsilon_{v}=\varepsilon_{v p}+\varepsilon_{x r} \\
\varepsilon_{C}=\frac{1-\varepsilon_{v}}{1+f \frac{\rho_{C}}{\rho_{P t}}+f_{I C} \frac{\rho_{C}}{\rho_{N}}} \\
\varepsilon_{N}=f_{I C} \frac{\rho_{C}}{\rho_{N}} \varepsilon_{C} \\
\varepsilon_{P t}=f \frac{\rho_{C}}{\rho_{P t}} \varepsilon_{C}
\end{gathered}
$$

where $f_{p m}$ is the ratio of the volume of pores smaller than $20 \mathrm{~nm}$ to total volume of pores, $\varepsilon_{v p}$ is the volume fraction of primary pores, $\varepsilon_{x r}$ is the volume fraction of secondary pores obtained from nano-CT, $\varepsilon_{v}$ is the calculated total porosity of the subsample, $\varepsilon_{C}, \varepsilon_{N}$, and $\varepsilon_{P t}$ are the 
volume fractions of $\mathrm{C}$, ionomer, and Pt phases, respectively, and $\rho_{C}, \rho_{N}$, and $\rho_{P t}$ are the densities of $\mathrm{C}$, ionomer, and $\mathrm{Pt}$ phases, respectively.

The solid phase observed in the nano-CT data corresponds to the well-known agglomerate structure which consists of $\mathrm{Pt} / \mathrm{C}$ aggregates, ionomer binder, and primary pores. To mimic this structure with a numerical reconstruction algorithm, it is assumed that the agglomerates were formed based on the clustering of primary $\mathrm{C}$ particles. In addition, the primary support particles and the catalyst particles are assumed to be ideal spheres. With inspiration from the QSGS algorithm [38], which is shown to be successful for porous media generation, the $\mathrm{C}$ phase is grown in the nano-CT extracted solid volume. Following the $\mathrm{C}$ phase growth, Pt and ionomer phases are generated by considering the interactions between C-Pt and C-Pt-ionomer. It should be noted that the primary pores are naturally formed as a consequence of this process.

The reconstruction procedure, illustrated in Fig. 3, is summarized below.

a) The nano-CT extracted $1 \mu \mathrm{m} \times 1 \mu \mathrm{m} \times 1 \mu \mathrm{m}$ solid volume, Fig. $2 \mathrm{a}$, which consists of $20 \mathrm{~nm}$ voxels, is mapped into a $2.5 \mathrm{~nm}$ voxel size domain with 400 x 400 x 400 computational cells, as shown in Fig. 3a.

b) The core particles for the growing $\mathrm{C}$ phase are randomly located within the solid geometry based on a distribution probability ' $c_{p c}$ ' and the size distribution of primary C particles, as shown in Fig. 2d. The distribution probability corresponds to the amount and dispersion of primary $\mathrm{C}$ particles that are needed to be placed as cores for the growing phase, as in QSGS algorithm. The primary C particle size distribution pool is generated from the TEM data in Fig. 2d. Looping through the unit cells in the 
solid phase geometry, a candidate primary $\mathrm{C}$ particle center is first determined with the help of a random generator by using $c_{p c}$ as the threshold value. Next, the primary C particle size is randomly determined from the size distribution pool, and avoiding overlapping particles, the core particles are assigned size within one loop over all the cells in the solid phase.

c) A percolation $\mathrm{C}$ network is grown into the nano-CT solid volume by looping through the existing particles and connecting them to fit their neighborhood, as in Fig. 3c. For each existing support particle, $A_{i}$, a candidate particle size is randomly picked from the size distribution pool. Then, the distance $d_{C}$ between the centers of candidate particle and $A_{i}$ is calculated by forcing them to touch. All the unit cells within $d_{C}$ distance from the center of $A_{i}$ are searched to be the center of the candidate particle. Next, by looping through the candidate $\mathrm{C}$ particle center cells, it is checked if there is any other $\mathrm{C}$ particle neighbors except $A_{i}$. If so, that cell is assigned to be the center and, if not, one of the center candidates is randomly picked and the spherical support is generated. This process is repeated until the desired volume fraction is achieved.

d) Catalyst particles are randomly located on the surface of the support particles based on the size distribution obtained from TEM, as in Fig. 3d. As in step (c), for each support particle, $A_{i}$, a candidate Pt size is picked from the size distribution pool binned to the model grid size, shown in Fig. 2f. The distance $d_{P t}$ between $A_{i}$ and the candidate catalyst particle center is calculated by forcing them to touch. Then one of the available unit cells in $d_{P t}$ distance from the center of $A_{i}$ is randomly selected as the cell center. The catalyst particle with given size is generated at that center. This process is applied until the required volume fraction is obtained. 
e) The ionomer network is generated by randomly placing ionomer particles on the surface of $\mathrm{Pt} / \mathrm{C}$ particles and enlarging them in the neighborhood of the generated ionomer particles, as in Fig. 3e. Looping through $\mathrm{C}$, Pt, and generated ionomer particles, available neighboring points are determined and then the ionomer particles (in shape of $2.5 \mathrm{~nm}$ voxel) are placed by using ionomer volume fraction as the generation probability. This process is repeated until the desired ionomer volume fraction is obtained.

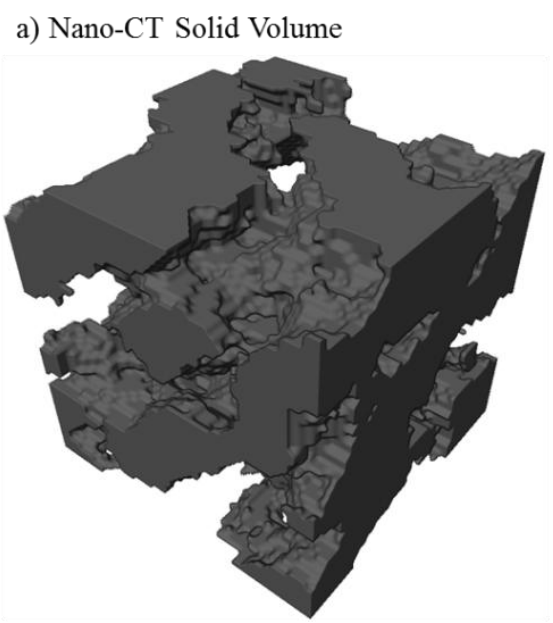

d) Place Pt on C Surface b) Core C Particles

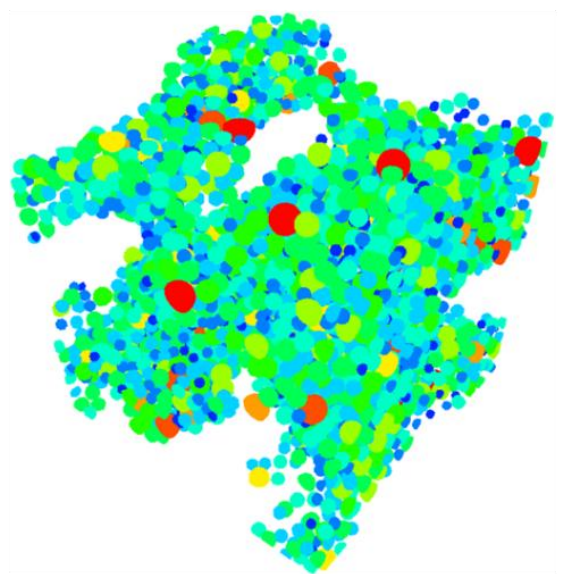

c) Grow C Network

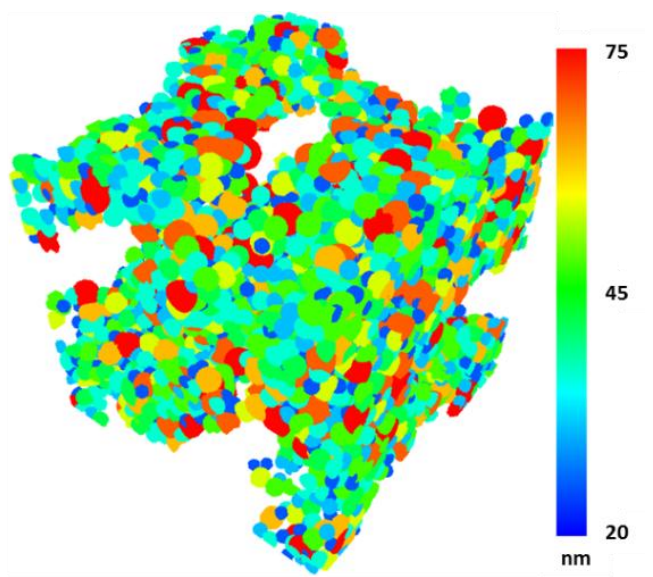

e) Generate Ionomer
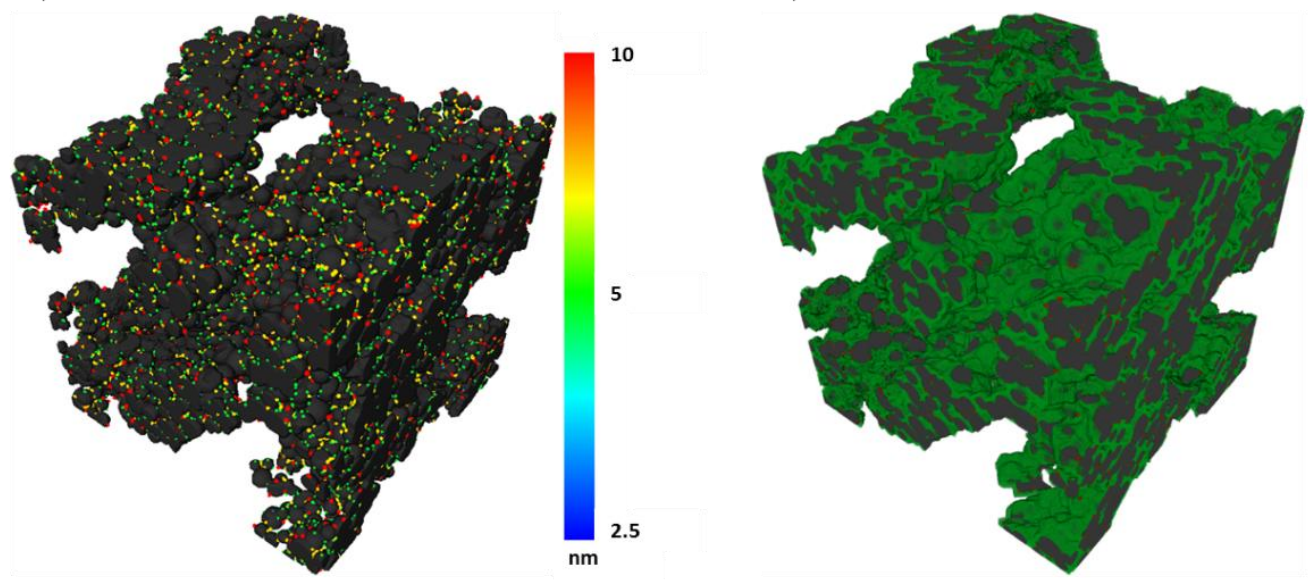

Figure 3. Description of hybrid reconstruction algorithm 
This technique focuses on the nanostructures within the secondary pore space and facilitates the study of smaller size scale physical phenomenon than those available given the current status of the resolution of the nano-CT technique. Therefore, depending on the dimensions of the generated structures, a $1 \mu^{3}$ domain size is considered to be representative of the physical characteristics below the resolution of nano-CT, such as the transport properties of primary pores, most of which are smaller than $10 \mathrm{~nm}$. The resulting microstructure is controlled by the morphology provided by nano-CT data, size distribution obtained from TEM data, the volume fraction of primary pores within the nano-CT solid volume determined based on porosimetry data and the distribution probability $\left(c_{p c}\right)$ that is used to locate the primary support particles as cores to be grown. Among all these, $c_{p c}$ is the only arbitrary controlling numerical parameter used in this method. Larger values of $c_{p c}$ would result in more closely packed $C$ support and the opposite for a smaller value. For the microstructure presented in this section, $c_{p c}=0.04$ is used and the porosity of the nano-CT sample increased from 41 to $45 \%$ with the addition of primary pores.

Determination of pore sizes is very important for microstructural characterization and for calculating electrode performance as it is directly related to reactant transport. The average chord length method [17] is employed to calculate the pore size distribution of the hybrid microstructure in Fig. 3e, which is used for validation of the hybrid microstructure. Since the secondary pore morphology is provided by the nano-CT technique, the focus of validation is the numerically-generated small pores. Distribution of pore sizes smaller than $40 \mathrm{~nm}$ are compared against MIP and BET data, as shown in Fig. 4a. Prior to comparison, it should be noted that the smallest pore sizes computed for BET, MIP, and hybrid are 1.35, 3, and $2.5 \mathrm{~nm}$, respectively. The BET technique is superior in detecting small size pores whereas it fails to capture pores 
bigger than $100 \mathrm{~nm}$ and the MIP and hybrid methods fail to resolve all the pores in the $0-10 \mathrm{~nm}$ size range which are captured by BET. Some of the small pores detected by BET may correspond to the pores in the Ketjen particles. The assumption of an ideal solid spherical shape for the primary support particle and the $2.5 \mathrm{~nm}$ resolution are the main limitations of the methodology. These limitations give rise to the discrepancy between the model and the BET primary pore size distributions. Besides that, the overall trend from the hybrid method agrees well with the results from both experimental techniques, with the hybrid method showing frequent pores in the $0-10 \mathrm{~nm}$ range followed by a decrease and then a gradual increase in pore frequency toward $40 \mathrm{~nm}$ size pores.

Quantitative analysis of the ionomer network is quite important, especially for optimization of the electrode microstructure. Direct imaging of the three-dimensional ionomer network inside the electrode still remains a challenge. Advanced TEM techniques have been used to image the ionomer via either microtoming and two-dimensional imaging of a series of slices or via three-dimensional imaging (ET) for a few ionomer-coated carbon particles [16, 17]. The sample preparation and application of these techniques is in its infancy, but will soon be able to provide important information on the three-dimensional distribution of ionomer. Recently, Babu et al. [23] reported 3D visualization of ionomer by employing nano-CT for thick PGM-free electrodes with the help of increased contrast due to $\mathrm{Cs}^{+}$staining. No size distribution for ionomer was provided in this study. Here it should be noted that besides the low contrast, the resolution of the nano-CT is another challenge for fully capturing the ionomer microstructure. None of the numerical characterization studies provide size distribution for ionomer since they are not detailed enough (i.e., ionomer is treated as a mixture with $\mathrm{C}$ and $\mathrm{Pt}$ [31, 32] or modeled as a uniform cover [33-35]). In this study, ionomer is generated within the nano-CT solid phase 
after the random agglomeration of $\mathrm{Pt} / \mathrm{C}$ particles according to the experimentally-obtained size distribution. The resulting three-dimensional ionomer network and the ionomer size distributions for four different random agglomeration and ionomer particle placements, which are calculated by using the sphere fitting method, are presented in Figs. $4 \mathrm{~b}$ and $4 \mathrm{c}$, respectively. To test the effect of the random placement of nanostructures, the ionomer size distribution of four microstructures generated by this technique for the same input parameters are compared in Fig. 4c. The similarity of these four distributions indicates that the experimentally-obtained parameters constrain the resulting ionomer size distributions and the generated microstructure. Here it is important to note that the presented size distribution does not directly correspond to the thickness of ionomer film (coverage) on the $\mathrm{C}$ particles because in some regions ionomer forms clusters, due to aggregation, in between separate $\mathrm{C}$ aggregates and blocks the pores, as can be seen in Fig. 4b and in the inset of Fig. 4c. Large sizes (15-30 nm) in Fig. 4c correspond to ionomer aggregates while the thickness of ionomer film covering the support particles is observed to range from 5 to $12.5 \mathrm{~nm}$. Agglomerates of ionomer, not forming thin films on the catalyst/carbon surface, have been found via cryogenic SEM of water-rich catalyst-ionomer inks [41]. 
a)

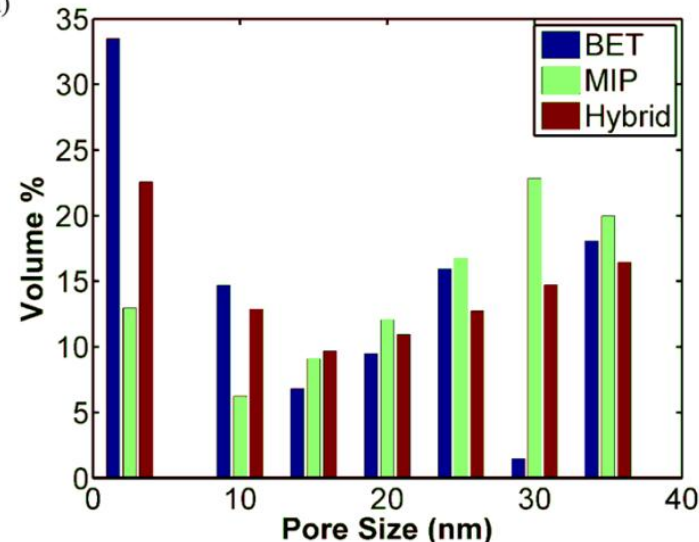

c)

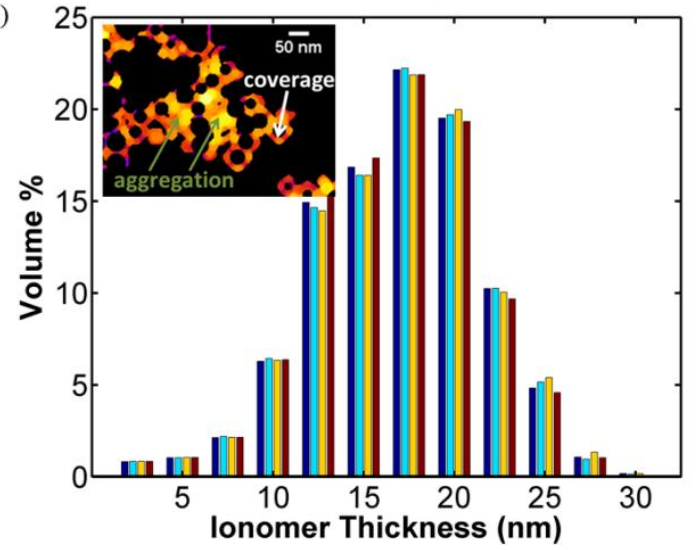

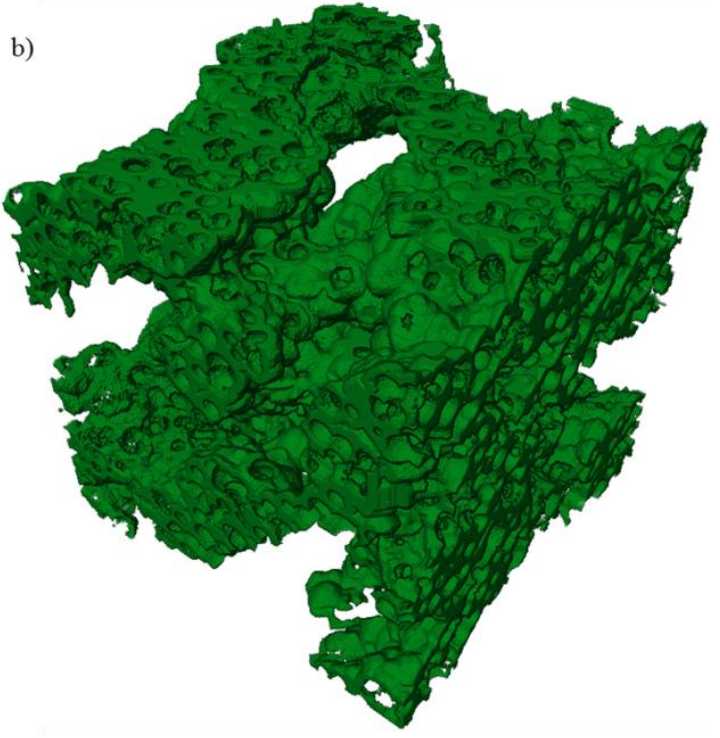

b)

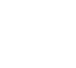

Figure 4. (a) Comparison of pore size distributions obtained with BET, MIP and hybrid approaches; (b) 3D ionomer network $(1 \mu \mathrm{m} \times 1 \mu \mathrm{m} \times 1 \mu \mathrm{m})$ generated with hybrid approach; (c) Ionomer thickness distribution for four microstructures generated by the hybrid method; inner plot shows the ionomer coverage on $\mathrm{C}$ support and aggregated ionomer particles

\section{Transport Simulations}

Multiple transport processes occur within the electrode microstructure during the PEMFC operation. It is crucial to understand the interactions between the microstructure and the transport processes. In this section, transport-related characteristic properties are investigated by performing simulations within the pore scale nano-CT geometry and the corresponding hybrid microstructure.

The porosity in the nano-CT data changes depending on the location and the size of the region of interest. The effect of local microstructure composition on the transport properties is 
investigated by extracting $0.5 \mu \mathrm{m}$ cubic subsamples, with porosities of $0.34,0.42,0.49$, from the nano-CT data. Subsequently, these subsamples are reconstructed with all the components by employing the hybrid approach presented in the previous section. The hybrid microstructures consist of $200 \times 200 \times 200$ unit cells. The connectivity of each phase (pore, C, and ionomer) needs to be determined to run the transport simulations. All the pore cells connected to a percolation network, which generates a path throughout the domain, are marked as active pore cells. In the absence of reactions, with the goal of calculating effective transport properties, reactant diffusion and the charge conduction is modeled by the divergence of flux vectors within the active transport cells, as illustrated in Figs. 5a, 5b, and 5c.

$$
\begin{aligned}
& \nabla \cdot \Gamma=0 \\
& \Gamma=\left\{\begin{array}{cc}
D_{t} \nabla c_{O_{2}}, & \text { for } O_{2} \\
\sigma_{m} \nabla \phi_{\mathrm{m}}, & \text { for } H^{+} \\
\sigma_{s} \nabla \phi_{\mathrm{s}}, & \text { for } e^{-}
\end{array}\right.
\end{aligned}
$$

where $D_{t}$ is the diffusivity coefficient for $\mathrm{O}_{2}$ transport, $c_{O_{2}}$ is the $\mathrm{O}_{2}$ concentration, $\sigma_{m}$ is the ion conductivity, $\phi_{\mathrm{m}}$ is the ion potential, $\sigma_{s}$ is the electron conductivity, $\phi_{\mathrm{s}}$ is the electron potential. In order to measure the flux accurately, extra layers of active cells were added to opposing faces of the domain and Dirichlet boundary conditions are assigned, as illustrated in Fig. 8. Periodic (no flux) boundary conditions are assigned at the remaining boundaries. All the governing equations are solved by using a commercial CFD code STAR CCM+ until the flux difference at the opposing faces becomes smaller than $10^{-6}$. It is necessary to mention that the transport coefficients are locally assigned instead of generating a surface mesh for the microstructure such that the whole computational domain is meshed and zero transport coefficients are assigned at the inactive cells. 

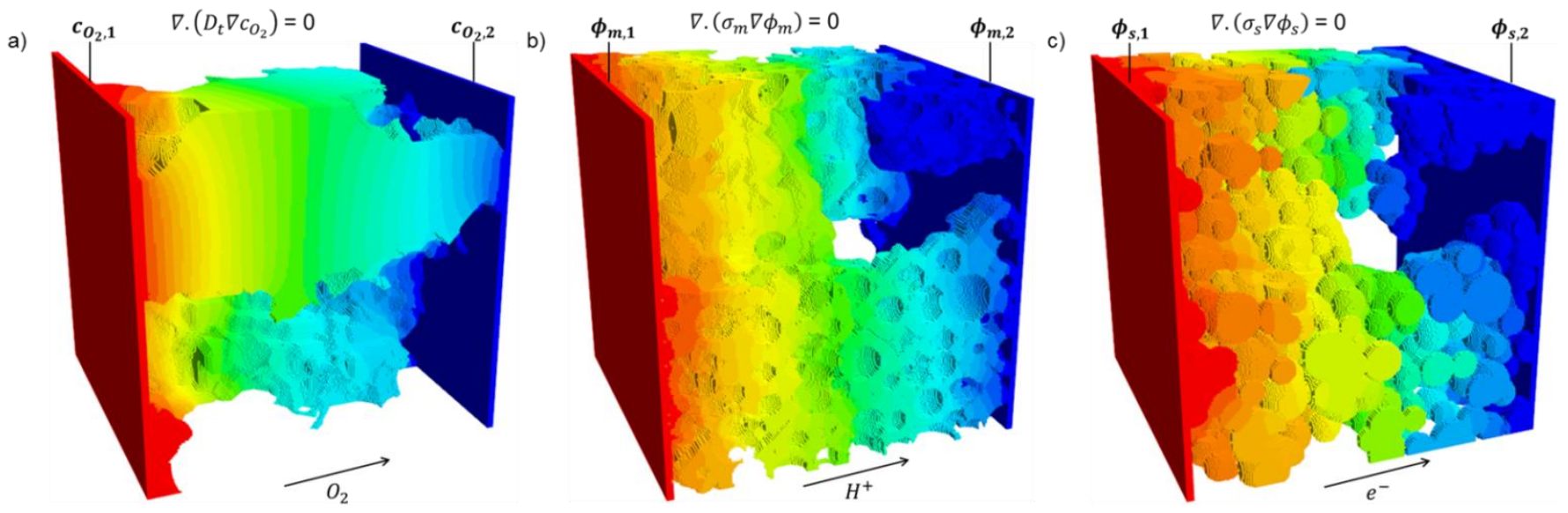

Figure 5. Description of computational domain and boundary conditions for (a) $\mathrm{O}_{2}$ transport; (b) $\mathrm{H}^{+}$ transport; (c) e- transport

The variation of $\mathrm{e}^{-}$and $\mathrm{H}^{+}$conductivities with respect to $\mathrm{C}$ and ionomer morphology is neglected. On the other hand, accounting for both Fickian and Knudsen diffusion regimes, local $\mathrm{O}_{2}$ diffusivity $\left(D_{t}\right)$ is computed by the Bosanquet formula as follows:

$$
\begin{gathered}
\frac{1}{D_{t}}=\frac{1}{D_{b}}+\frac{1}{D_{k}} \\
\mathrm{D}_{\mathrm{k}}=\frac{d_{p}}{3} \sqrt{\frac{8 R T}{\pi M_{O_{2}}}}
\end{gathered}
$$

where $D_{b}$ is the bulk diffusivity of $\mathrm{O}_{2}$ in air, $D_{k}$ is the Knudsen diffusivity, $d_{p}$ is the local pore diameter (in $\mathrm{m}$ ) calculated by the chord length method, and $\mathrm{T}$ is the temperature (in $\mathrm{K}$ ).

Depending on the flux computed by the simulations, effective transport coefficients $\left(D_{e f f}, \sigma_{m, e f f}, \sigma_{s, e f f}\right)$ are calculated by employing Fick's law:

$$
p_{e f f}=J \frac{t_{c l}}{\Delta c}
$$

where $p_{e f f}$ corresponds to $D_{e f f}, \sigma_{m, e f f}, \sigma_{s, e f f}$ respectively for $\mathrm{O}_{2}, \mathrm{H}^{+}$, e transport processes, $J$ is the flux computed at the inlet and outlet boundaries for each transport simulation, $t_{c l}$ is the thickness of the computational domain, $c$ corresponds to $c_{O_{2}}, \phi_{\mathrm{m}}, \phi_{\mathrm{s}}$ respectively for $\mathrm{O}_{2}, \mathrm{H}^{+}, \mathrm{e}^{-}$ 
transport processes. Once the effective transport coefficients are computed, tortuosity $(\tau)$ for each transport process is expressed as follows:

$$
\tau=\varepsilon_{\mathrm{i}} \frac{p_{\text {bulk }}}{p_{\text {eff }}}
$$

where $\varepsilon_{\mathrm{i}}$ corresponds to volume fractions of pores, ionomer, $\mathrm{Pt} / \mathrm{C}$ for $\mathrm{O}_{2}, \mathrm{H}^{+}$, $\mathrm{e}^{-}$transport processes, respectively, $p_{\text {bulk }}$ corresponds $\overline{D_{t}}, \sigma_{m}, \sigma_{s}$ for $\mathrm{O}_{2}, \mathrm{H}^{+}, \mathrm{e}^{-}$transport processes, respectively. Here it should be noted that $\overline{D_{t}}$ is the volume-averaged $\mathrm{O}_{2}$ diffusivity since $D_{t}$ changes locally.

\subsection{Results of Transport Simulations}

The microstructure reconstructed with the hybrid algorithm enables one to investigate detailed morphological and transport properties. In this section, transport properties of the hybrid microstructure are briefly presented.

Recalling that the nano-CT data and the hybrid microstructure share the same secondary pore morphology, the effect of primary pores on the reactant transport is investigated by running reactant transport simulations for both. Effective diffusivities for nano-CT data and the corresponding hybrid microstructure are computed as shown in Fig. 9a. Figure 9a shows that the effective diffusivities for the hybrid microstructure are slightly higher than the ones for nano-CT microstructure. The addition of the primary pores to the nano-CT domain lowers the tortuosity of the hybrid microstructure, as shown in the inset of Fig. 9a. Lower tortuosity of the hybrid microstructure is associated with the extra reactant transport pathways formed by the primary pores, as shown in Fig. 9b. On the other hand, local diffusivities within the extra pathways are very low due to dominant Knudsen effects in the primary pores, as illustrated in Fig. 9c. Therefore, addition of the primary pores results in minor increases of the effective diffusivities 
with respect to the nano-CT geometry. Here it should be noted that primary pores would play a more significant role for calculation of transport resistance from inlet to catalyst surface. This will be studied in future work where we investigate electrochemical reactions within the hybrid microstructure.

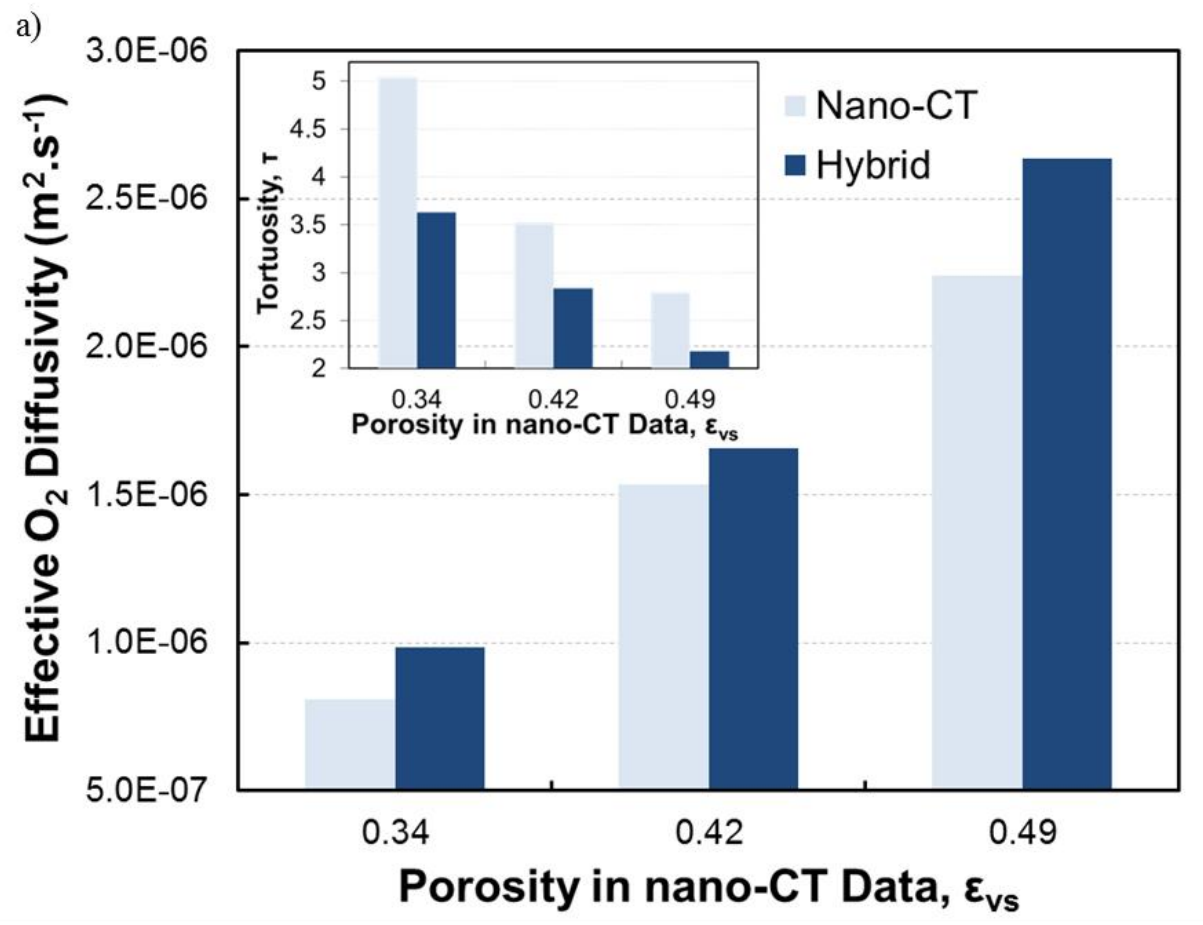

b)

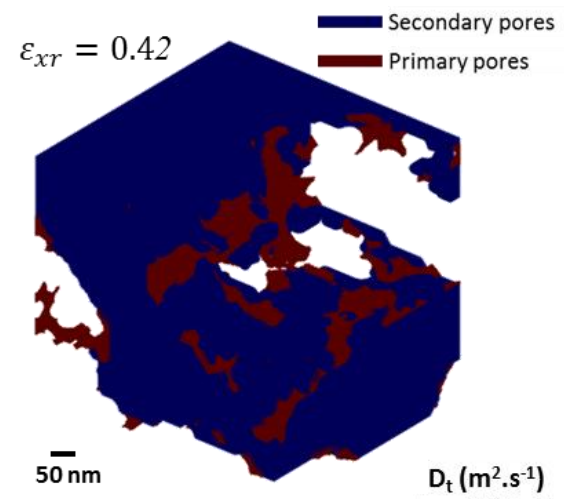

c)
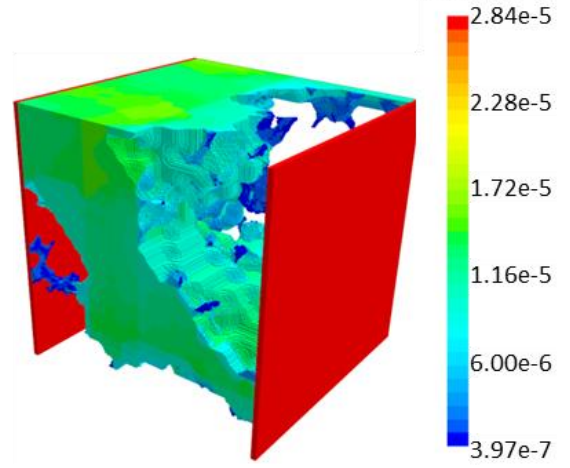

Figure 6. (a) Comparison of effective $\mathrm{O}_{2}$ diffusivities calculated for nano-CT data and the corresponding hybrid microstructure; the inner plot shows the corresponding tortuosity values; (b) Primary and secondary pore volumes for the hybrid microstructure corresponding to $42 \%$ nano-CT porosity; (c) Local $\mathrm{O}_{2}$ diffusivity for the microstructure shown in Fig. 6b 

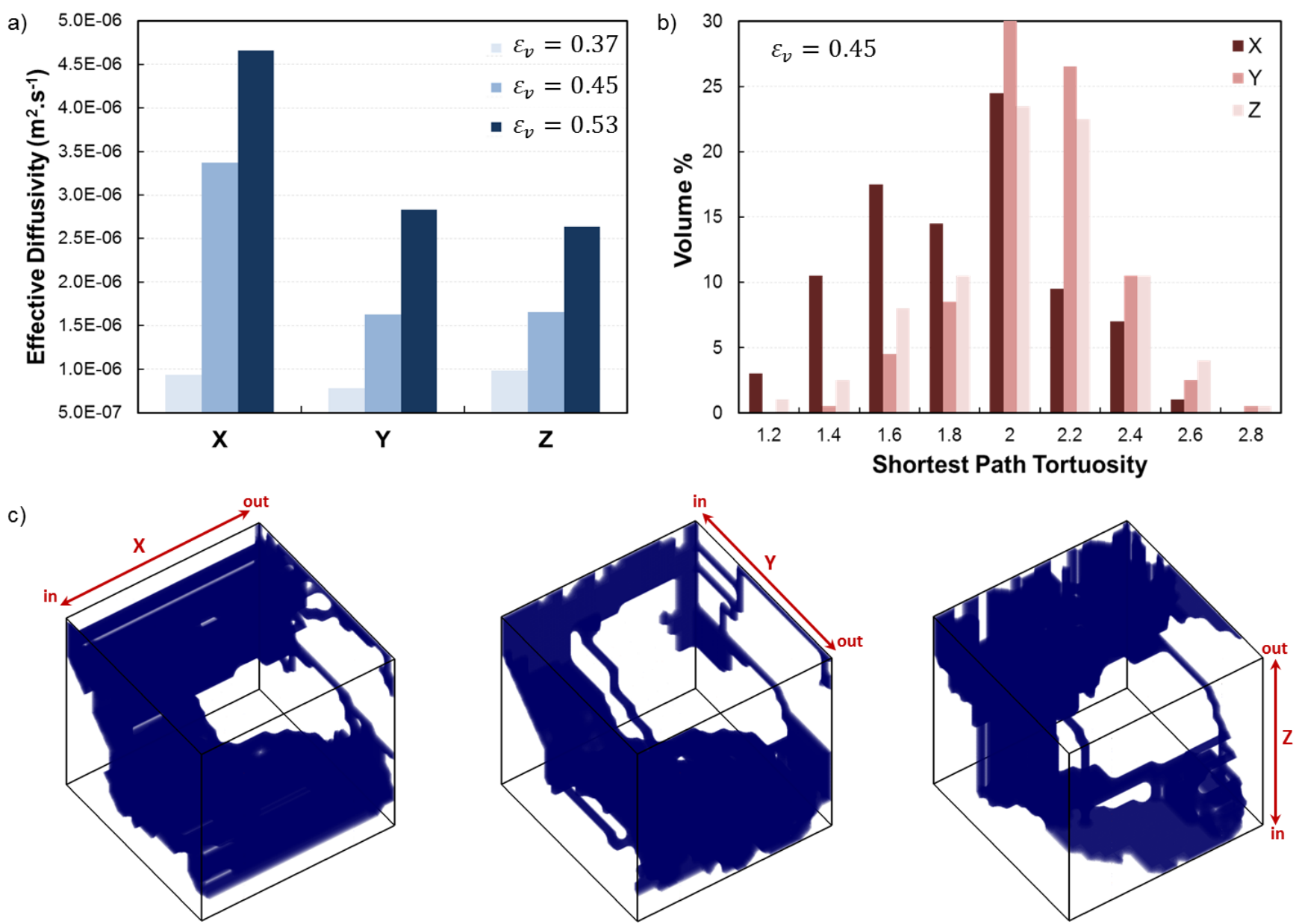

Figure 7. (a) Effective $\mathrm{O}_{2}$ diffusivities in orthogonal directions for hybrid microstructure; (b) Shortest path tortuosity in orthogonal directions in pore phase of nano-CT data corresponding to $\varepsilon_{v}=0.45$ hybrid microstructure; (c) Computed paths from the entry to exit in orthogonal directions

Reactant transport simulations were conducted separately in orthogonal directions ( $\mathrm{x}, \mathrm{y}$, and $\mathrm{z}$ ) within the hybrid microstructure. Figure 7 a shows the computed effective diffusivities in $\mathrm{x}, \mathrm{y}$, and $\mathrm{z}$ directions for subsamples with different porosities. For the 45 and $53 \%$ porosity samples, one can see that effective diffusivities in the $\mathrm{x}$ direction are higher than those in the $\mathrm{y}$ and $\mathrm{z}$ directions. On the other hand, for the sample with $37 \%$ porosity, the highest effective diffusivity is obtained in the $\mathrm{z}$ direction. The anisotropy of microstructure is also investigated by calculating the shortest path tortuosity [22] in orthogonal directions for the nano-CT sample corresponding to the hybrid microstructure with $45 \%$ porosity. Figure $7 \mathrm{~b}$ and $7 \mathrm{c}$ show the 
shortest path tortuosity and the computed paths, respectively. Figure $7 \mathrm{~b}$ indicates lower shortest path tortuosity for the $\mathrm{x}$ direction in comparison to the $\mathrm{y}$ and $\mathrm{z}$ directions. This can also be visually observed in Fig. 7c, which shows the reactant pathways from inlet to outlet are almost straight in the $\mathrm{x}$ direction unlike the $\mathrm{y}$ and $\mathrm{z}$ directions. So the high effective diffusivity with the $45 \%$ porosity hybrid microstructure in the $\mathrm{x}$ direction with respect to other directions can be associated with the shortest path tortuosity imposed by the nano-CT geometry. This result also shows the dominant effect of nano-CT data on the reactant transport simulations. Overall Fig. 7 shows the random anisotropy and the heterogeneity with in the electrode microstructure.

Next, charge transport is investigated by performing simulations within the generated ionomer and $\mathrm{C}$ networks in the hybrid microstructure. Figure $8 \mathrm{a}$ and $8 \mathrm{~b}$ show the computed relative conductivities, which correspond to the ratio of the effective ionomer conductivity to bulk conductivity, along with the tortuosity values for $\mathrm{H}^{+}$and $\mathrm{e}^{-}$transport, respectively. Figure 8a indicates that the effective ion conductivity increases with decreasing porosity because of corresponding increase in the ionomer volume fraction and size. As a natural consequence of the increasing ionomer volume fraction, more pathways are generated for ion transport and the tortuosity decreases, as shown in the inset of Fig. 8a. Similarly Fig 8b shows that the effective electron conductivity is improved as the porosity decreases due to increasing volume fraction of C phase. 
a)

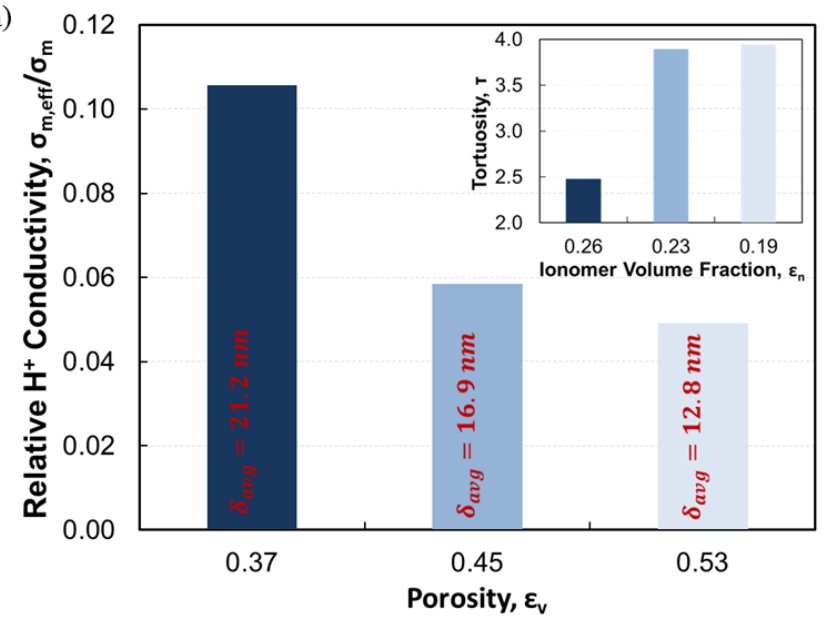

c)

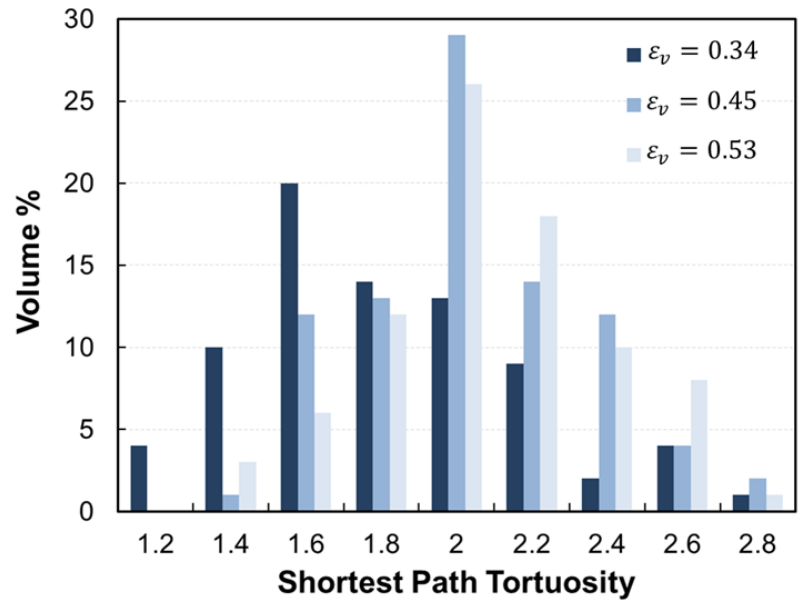

b)

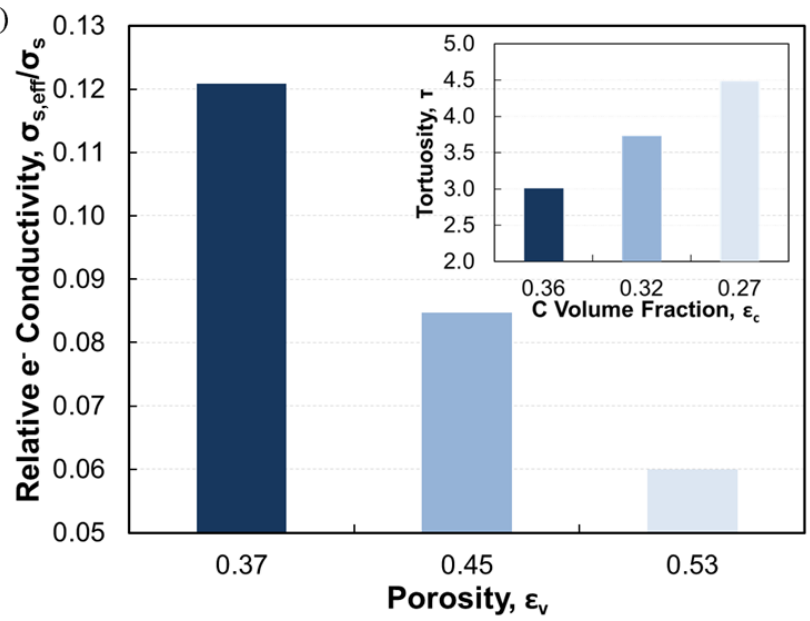

d)

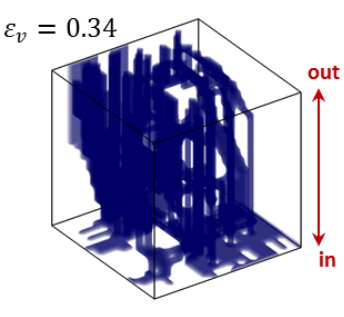

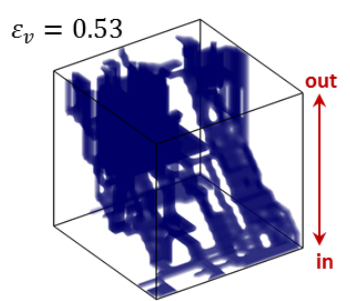

Figure 8. (a) Relative $\mathrm{H}^{+}$conductivity within reconstructed ionomer network ( $\delta_{\text {avg }}$ is the average ionomer size) and the corresponding tortuosity in the inner set; (b) Relative e ${ }^{-}$conductivity within reconstructed $\mathrm{C}$ network and the corresponding tortuosity in the inner set; (c) Shortest path tortuosity for in X-CT solid phase corresponding to hybrid microstructures in Fig. 11; (d) Corresponding computed paths from the entry to exit

It is interesting that there is a dramatic change of tortuosity between 23 and $26 \%$ ionomer volume fraction microstructures in comparison to the slight change of tortuosity between 19 and $23 \%$, as shown in the inset of Fig. 8a. The shortest path tortuosity of the solid volume in the nano-CT sample is computed, as shown in Fig. 8c and 8d. Figure 8c shows that the shortest path tortuosity values corresponding to the $34 \%$ porosity sample are low, whereas the values for the other two samples are quite close to each other following the trend in the inset of Fig. 8a. Apparently, the tortuosity of ionomer in the generated ionomer network is strongly affected by 
the tortuosity of the solid phase in the nano-CT data. On the other hand, the effect of the nanoCT solid phase tortuosity is not reflected in the electron transport simulations. So it can be deduced that the reconstructed $\mathrm{C}$ network overwhelms the effect of the nano-CT geometry, unlike that of the ionomer network. This can be explained by the size difference between the ionomer and the $\mathrm{C}$ particles.

\section{Summary and Conclusions}

Incorporating different experimental techniques, a hybrid approach is presented to reconstruct the detailed PEMFC electrode microstructure. Nano-CT was performed to extract the morphology of the secondary pore space. TEM images, X-ray scattering, and porosimetry techniques were used to obtain statistical information about the nano scale structures within the electrode. Information from these different sources were combined with an algorithm proposed in this study, to regenerate the electrode microstructure from primary $\mathrm{C}, \mathrm{Pt}$, and ionomer particles within the nano-CT extracted volume. The microstructure was validated by comparing the pore size distribution against the porosimetry data.

The ionomer microstructure plays a crucial role in electrodes as it impacts the ion conduction, reactant transport, and catalyst utilization. Even though it is an approximation, this approach produces a realistic ionomer microstructure and primary pores unlike previous approaches utilized for electrode microstructure generation [31-37]. To the best of the authors' knowledge, ionomer size distribution is calculated for the first time by combining nano-CT with experimental and numerical techniques.

Unlike previous imaging attempts [19-22] this technique allows the study of the effects of primary pores on reactant transport. Performing transport simulations for both nano-CT and 
hybrid microstructures, it is observed that addition of primary pores decreases tortuosity but has a minor effect on the computed effective diffusivity due to an augmented Knudsen effect in the small pores. On the other hand, in the case of calculating the transport resistance to the catalyst particle surface, the primary pores would play a significant role. This effect will be incorporated in future studies where the hybrid microstructure will be employed in a multiphysics model accounting for electrochemical reactions. This will also aid in understanding catalyst utilization under different operating regimes and in the improving electrode design with lower Pt loading. In addition to the effects of primary pores on effective reactant transport properties, probing the electrode microstructure with transport simulations and shortest path tortuosity computations, it can be concluded that pore morphology is heterogeneous and anisotropic in agreement with Litster et al. [18, 22].

With its ability to approximate the $\mathrm{C}$ and ionomer microstructures individually, the hybrid microstructure facilitates characterization of the effective $\mathrm{e}^{-}$and $\mathrm{H}^{+}$transport properties. In addition to the expected trend of improved effective conductivities with increased volume fractions of conductive phases, it can be concluded that the effective $\mathrm{H}^{+}$conduction properties are highly dependent on the electrode morphology, as determined by nano-CT, due to the small size of ionomer nanostructures.

\section{Acknowledgements}

This work is part of a collaborative project with Johnson Matthey Fuel Cells, United Technologies Research Center, the University of Texas-Austin, and Indiana University-Purdue University of Indianapolis. The authors wish to thank the U.S. Department of Energy's Fuel Cell Technologies Office (Nancy Garland, program manager) for support. This research used 
resources of the Advanced Photon Source (APS), a U.S. Department of Energy (DOE) Office of Science User Facility operated for the DOE Office of Science by Argonne National Laboratory under Contract No. DE-AC02-06CH11357. Argonne National Laboratory is managed for the U.S Department of Energy by the University of Chicago Argonne, LLC, also under contract DEAC-02-06CH11357.

\section{Nomenclature}

\begin{tabular}{|c|c|}
\hline $\mathrm{C}_{\mathrm{O}_{2}}$ & $\mathrm{O}_{2}$ concentration, mol. $\mathrm{m}^{-3}$ \\
\hline $\mathrm{D}_{\mathrm{b}}$ & bulk $\mathrm{O}_{2}$ diffusivity in air, $m^{2} \cdot s^{-1}$ \\
\hline$D_{\text {eff }}$ & effective $\mathrm{O}_{2}$ diffusivity, $\mathrm{m}^{2} \cdot \mathrm{s}^{-1}$ \\
\hline $\mathrm{D}_{\mathrm{k}}$ & local Knudsen diffusivity for $\mathrm{O}_{2}, \mathrm{~m}^{2} . \mathrm{s}^{-1}$ \\
\hline $\mathrm{D}_{\mathrm{t}}$ & local $\mathrm{O}_{2}$ diffusivity calculated by Bosanquet formula, $\mathrm{m}^{2} . \mathrm{s}^{-1}$ \\
\hline$d_{p}$ & local pore size, $m$ \\
\hline $\mathrm{f}$ & weight ratio of $\mathrm{Pt}$ to $\mathrm{C}$ \\
\hline$f_{p m}$ & ratio of primary pore volume to total pore volume \\
\hline$f_{I C}$ & weight ration of ionomer to $\mathrm{C}$ \\
\hline$J$ & flux calculated at the inlet and outlet boundaries, mol. $\mathrm{m}^{-2} \mathrm{~s}^{-1}$ \\
\hline$M_{O_{2}}$ & molecular weight of $\mathrm{O}_{2}, \mathrm{~kg} \cdot \mathrm{mol}^{-1}$ \\
\hline$R$ & ideal gas constant, $j . \mathrm{mol}^{-1} \mathrm{~K}^{-1}$ \\
\hline$T$ & temperature, $K$ \\
\hline$t_{c l}$ & thickness of the sample, $m$ \\
\hline$T$ & temperature, $K$ \\
\hline$\Gamma$ & flux vector, mol. $m^{-2} s^{-1}$ \\
\hline$\varepsilon_{C}$ & $\mathrm{C}$ volume fraction \\
\hline$\varepsilon_{N}$ & ionomer volume fraction \\
\hline$\varepsilon_{P t}$ & Pt volume fraction \\
\hline$\varepsilon_{v}$ & pore volume fraction \\
\hline$\varepsilon_{v p}$ & volume fraction of primary pores \\
\hline$\varepsilon_{x r}$ & volume fraction of secondary pores from $\mathrm{X}-\mathrm{CT}$ \\
\hline$f_{I C}$ & weight ration of ionomer to $\mathrm{C}$ \\
\hline$\rho_{C}$ & density of $\mathrm{C}, \mathrm{kg} \cdot \mathrm{m}^{-3}$ \\
\hline$\rho_{N}$ & density of ionomer, $\mathrm{kg} \cdot \mathrm{m}^{-3}$ \\
\hline$\rho_{P t}$ & density of $\mathrm{Pt}, \mathrm{kg} \cdot \mathrm{m}^{-3}$ \\
\hline$\sigma_{m}$ & $\mathrm{H}^{+}$conductivity, $S . m^{-1}$ \\
\hline$\sigma_{m, e f f}$ & effective $\mathrm{H}^{+}$conductivity, $S . m^{-1}$ \\
\hline$\sigma_{s}$ & $\mathrm{e}^{-}$conductivity, $S \cdot m^{-1}$ \\
\hline$\sigma_{s, e f f}$ & effective $\mathrm{e}^{-}$conductivity, $S . m^{-1}$ \\
\hline
\end{tabular}


$\begin{array}{ll}\phi_{m} & \mathrm{H}^{+} \text {potential, } V \\ \phi_{s} & \mathrm{e}^{-} \text {potential, } V\end{array}$

\section{References}

1. USDRIVE, Fuel Cell Technical Team Roadmap, June 2013

2. M. Uchica, Y. Aoyama, N. Eda, A. Ohta, Journal of Electrochemical Society,142 (1995) 4143

3. E. Antolini, L. Giorgi, A. Pozio, E. Passalacqua, Journal Power Sources, 77 (1999) 136

4. V.A. Paganin, E.A. Ticinalli, E.R. Gonzalez, Journal of Applied Electrochemistry, 26 (1996) 297

5. E. Passalacqua, F. Lufrano, G. Squadrito, A. Patti, L. Giorgi, Electrochimica Acta, 46 (2001) 799

6. Z. Qi, A. Kaufman, Journal of Power Sources, 113 (2003) 37

7. G. Sasikumar, J.W. Ihm, H. Ryua, Journal of Power Sources, 132 (2004) 11

8. T. Sobolyeva, X. Zhao, X. Malek, Z. Xie, T. Navessin, S. Holdcroft, ACS Applied Materials \& Interfaces, 2 (2010) 375

(9) M. Chatenet, L. Guetaz, F. Maillard, Electron Microscopy to Study Membrane Electrode Assembly (MEA) Materials and Structure Degradation, in Handbook of Fuel Cells; John Wiley \& Sons, Ltd.: Chichester, UK, 2009; Vol. 6, pp 844

10. J. Xie, K.L. More, T.A. Zawodzinski, Journal of Electrochemical Society, 151 (11) (2004) A1841 
11. J. Xie, D.L. Wood, K.L. More, P. Atanassov, R.L. Borup, Journal of Electrochemical Society, 152 (5) (2005) A1011

12. P.J. Ferreira, G.J. la O’, Y. Shao-Horn, D. Morgan, R. Makharia, S. Kocha, H. A. Gasteiger, Journal of Electrochemical Society, 152 (11) (2005) A2256

13. P.J. Ferreira and Y. Shao-Horn, Electrochemical and Solid-State Letters, 10 (3) (2007) B60

14. E. Guilminot, A. Corcella, F. Charlot, F. Maillard, M. Chatenet, Journal of Electrochemical Society, 154 (1) (2007) B96

15. J. Xie, F. Xu, D.L. Wood III, K.L. More, T.A. Zawodzinski, W.H. Smith, Electrochimica Acta, 55 (2010) 7404

16. D.A. Cullen, R. Koestner, R.S. Kukreja, Z.Y. Liu, S. Minko, O. Trotsenko, A. Tokarev, L. Guetaz, H.M. Meyer III, C.M. Parish, K.L. More, Journal of Electrochemical Society, 161 (10) (2014) F1111

17. M. Lopez-Haro, L. Guetaz, T. Printemps, A. Morin, S. Escribano, P.-H. Jouneau, P. BayleGuillemaud, F. Chandezon, G. Gebel, Nature Communications, 5 (2014) 5229

18. W.K. Epting, J.Gelb, S. Litster, Advanced Functional Materials, 22 (2012) 555

19. C. Ziegler, S. Thiele, R. Zengerle, Journal of Power Sources, 196 (2011) 2094

20. S. Thiele, R. Zengerle, C. Ziegler, Nano Research, 4 (9) (2011) 849

21. S. Vierrath, F. Güder, A. Menzel, M. Hagner, R. Zengerle, M. Zacharias, S. Thiele, Journal of Power Sources, 285 (2015) 413 
22. S. Litster, W.K. Epting, E.A. Wargo, S.R. Kalidindi, E.C. Kumbur, Fuel Cells, 13 (2013) 935

23. S.K. Babu, H.T. Chung, P. Zelenay and S. Litster, ACS Applied Materials and Interfaces, 8 (2016) 32764

24. P.R. Shearing, R.S. Bradley, J. Gelb, S.N. Lee, A. Atkinson, P.J. Withers, N.P. Brandona, Electrochemical and Solid-State Letters, 14 (10) (2011) B117

25. W. Sun, B.A. Peppley, K. Karana, Electrochimica Acta, 50 (2005) 3359

26. M. Secanell, K. Karan, A. Suleman, N. Djilali, Electrochimica Acta, 52 (2007) 6318

27. W.K. Epting, S. Litster, International Journal of Hydrogen Energy, 37 (2012) 8505

28. S. Kamarajugadda, S. Mazumder, Journal of Power Sources 208 (2012) 328

29. F.C. Cetinbas, S.G. Advani, A.K. Prasad, Journal of Power Sources, 250 (2014) 110

30. F.C. Cetinbas, S.G. Advani, A.K. Prasad, Journal of Electrochemical Society, 161 (6) (2014) F803

31. P.P. Mukherjee, C.Y. Wang, Journal of Electrochemical Society, 153 (2006) A840

32. G. Wang, P.P. Mukherjee, C-Y. Wang, Electrochimica Acta, 52 (2007) 6367

33. S. H. Kim and H. Pitsch, Journal of Electrochemical Society, 156 (2009) 673

34. K.J. Lange, P-C. Sui, N.Djilali, Journal of Electrochemical Society, 157 (2010) 1434

35. K.J. Lange, P-C. Sui and N.Djilali, J. Power Sources 208 (2012) 354- 365

36. J. Zhang, W.Yang, L. Xu, Y. Wang, Electrochimica Acta 56 (2011) 6912- 6918 
37. N.A. Siddique, F. Liu, Electrochimica Acta, 55 (2010) 5357

38. M. Wang, J. Wang, N. Pan, S. Chen, Physical Review E, 75 (2007)

39. J. Ilavsky, P.R. Jemian, A.J. Allen, F. Zhang, L.E. Levine and G.G. Long. Journal of Applied Crystallography, 42 (2009) 469

40. J. Ilavsky, P.R. Jemian, Journal of Applied Crystallography, 42 (2009) 347

41. S. Takahashi, T. Mashio, N. Horibe, K. Akizuki, A. Ohma, ChemElectroChem, 2 (2015) 1560

\section{Figure Captions}

Figure 1. Representation of the principal components of the cathode electrode and their functions

Figure 2. (a) Nano-CT volume after segmentation to pore (void) and the solid phase (C, Pt, ionomer) to be used in the hybrid approach; (b) Corresponding pore size distribution; (c) TEM image of annealed Ketjen 300J C in powder; (d) Size distribution of primary C particles by TEM and USAXS; (e) TEM image of Pt on annealed C; (f) Size distribution of Pt particles from SAXS and TEM image analysis. The inset shows the size distribution of Pt particles from TEM image analysis binned to size of the modeling grid

Figure 3. Description of hybrid reconstruction algorithm

Figure 4. (a) Comparison of pore size distributions obtained with BET, MIP and hybrid approaches; (b) 3D ionomer network $(1 \mu \mathrm{m} \times 1 \mu \mathrm{m} \times 1 \mu \mathrm{m})$ generated with hybrid approach; (c) Ionomer thickness distribution; inner plot shows the ionomer coverage on $\mathrm{C}$ support and aggregated ionomer particles

Figure 5. Description of computational domain and boundary conditions for (a) $\mathrm{O}_{2}$ transport; (b) $\mathrm{H}^{+}$ transport; (c) e- transport

Figure 6. (a) Comparison of effective $\mathrm{O}_{2}$ diffusivities calculated for nano-CT data and the corresponding hybrid microstructure; the inner plot shows the corresponding tortuosity values; (b) Primary and secondary pore volumes for the hybrid microstructure corresponding to $42 \%$ nano-CT porosity; (c) Local $\mathrm{O}_{2}$ diffusivity for the microstructure shown in Fig. 6b

Figure 7. (a) Effective $\mathrm{O}_{2}$ diffusivities in orthogonal directions for hybrid microstructure; (b) Shortest path tortuosity in orthogonal directions in pore phase of nano-CT data corresponding to $\varepsilon_{v}=0.45$ hybrid microstructure; (c) Computed paths from the entry to exit in orthogonal directions 
Figure 8. (a) Relative $\mathrm{H}^{+}$conductivity within reconstructed ionomer network $\left(\delta_{\text {avg }}\right.$ is the average ionomer size) and the corresponding tortuosity in the inner set; (b) Relative $\mathrm{e}^{-}$conductivity within reconstructed $\mathrm{C}$ network and the corresponding tortuosity in the inner set; (c) Shortest path tortuosity for in X-CT solid phase corresponding to hybrid microstructures in Fig. 11; (d) Corresponding computed paths from the entry to exit

\section{Table Captions}

Table 1. Experimental characterization techniques and the obtained data 
Table 1. Experimental characterization techniques and the obtained data

\begin{tabular}{ll}
\hline Technique & Data \\
\hline Nano-CT & Pore network with 20 nm voxel size \\
TEM & Size distribution of primary C and Pt particles \\
USAXS & Complemantary data for size distribution of \\
MIP & primary C and Pt particles \\
BET & Pore volume size distribution $(3 \mathrm{~nm}-1 \mu \mathrm{m})$ \\
\hline
\end{tabular}

